\title{
Oncogenic WIP1 phosphatase attenuates the DNA damage response and sensitizes p53 mutant Jurkat cells to apoptosis
}

\author{
MEHTAP KILIC EREN $^{1 *}$, NUR BETÜL KARTAL ${ }^{2,4^{*}}$ and HATICE PILEVNELI ${ }^{3}$ \\ ${ }^{1}$ Department of Medical Biology, Faculty of Medicine; Departments of ${ }^{2}$ Medical Biochemistry and ${ }^{3}$ Medical Biology, \\ Institute of Health Sciences, University of Aydin Adnan Menderes, 09010 Aydin, Turkey
}

Received December 23, 2020; Accepted March 25, 2021

DOI: $10.3892 / \mathrm{ol} .2021 .12740$

\begin{abstract}
Wild-type (wt) p53-induced phosphatase 1 (Wip1), encoded by the protein phosphatase, $\mathrm{Mg}^{2+} / \mathrm{Mn}^{2+}$ dependent 1D (PPM1D) gene, is a serine/threonine phosphatase induced upon genotoxic stress in a p53-dependent manner. Wip1/PPM1D is frequently overexpressed, amplified and mutated in human solid tumors harboring wt p53 and is thus currently recognized as an oncogene. Oncogenic Wip1 dampens cellular stress responses, such as cell cycle checkpoints, apoptosis and senescence, and consequently increases resistance to anticancer therapeutics. Targeting Wip1 has emerged as a therapeutic strategy for tumors harboring wt p53. However, little is known about the efficacy of Wipl-targeted therapies in tumors lacking $\mathrm{p} 53$. The present study aimed to investigate the potential role of oncogenic Wip1 in p53 mutant (mt) Jurkat cells. In the present study, it was demonstrated that p53 mt Jurkat cells exhibited PPM1D/Wip1 gene amplification and expressed relatively high levels of Wip1, as confirmed by gene copy number and RNA expression analysis. In addition, Jurkat cells underwent G2 cell cycle arrest, apoptotic cell death and senescence in response to etoposide and doxorubicin, although the phosphorylation levels of DNA damage response (DDR) elements, including ataxia-telangiectasia mutated, ataxia-telangiestasia and Rad3-related, checkpoint kinase (Chk)1 and Chk2 were significantly low. Accordingly, the targeting of Wipl phosphatase by RNA interference increased the phosphorylation of DDR elements, but decreased the rate of apoptosis in response to etoposide or doxorubicin in Jurkat cells. The induction
\end{abstract}

Correspondence to: Dr Mehtap Kilic Eren, Department of Medical Biology, Faculty of Medicine, University of Aydin Adnan Menderes, Efeler, 09010 Aydin, Turkey

E-mail:mkilic@adu.edu.tr

${ }^{*}$ Contributed equally

Present address: ${ }^{4}$ Department of Medical Services and Techniques, Health Services Vocational School, 9 Eylül University, Balcova, 35330 İzmir, Turkey

Key words: p53-induced phosphatase 1, p53, Jurkat, senescence, apoptosis, etoposide, doxorubicin of senescence or cell cycle arrest was not affected by the knockdown of Wip1. The results suggest that increased Wip1 expression enhances the apoptotic sensitivity of Jurkat cells in response to chemotherapeutic agents by attenuating DDR signaling. The present study highlights the possible pro-apoptotic role of Wip1 in a p53 mt T-cell acute lymphoblastic leukemia cell line. The data suggest the careful consideration of future treatment strategies aiming to manipulate or target Wip1 in human cancers lacking p53.

\section{Introduction}

Eukaryotic cells have a conserved DNA damage response (DDR), which comprises cell cycle checkpoints, DNA repair, apoptosis and senescence, in order to prevent the transfer of damaged DNA to the next generations. In healthy cells, the DDR not only acts as a cellular response against DNA damage, but also as an intrinsic barrier against tumorigenesis (1). Therefore, the disruption of key factors involved in maintenance of the DDR may result in the initiation of tumor formation.

The DDR is activated by a large signaling network cascade controlled by the phosphorylation of ataxia-telangiectasia mutated (ATM) and ataxia-telangiestasia and Rad3-related (ATR) kinases, and checkpoint kinase (Chk)1 and Chk2, which are implicated in the activation of tumor suppressor protein $\mathrm{p} 53$. When activated, p53 induces the activation of p21, which inhibits cell cycle progression, thus contributing to the temporal arrest of the cell cycle and the initiation of DNA repair $(2,3)$. The DDR is terminated by serine/threonine phosphatases, allowing the recovery of checkpoints and the renewal of cell proliferation following DNA repair. Wild-type (wt) p53-induced phosphatase 1 (Wip1) is a member of the protein phosphatase $2 \mathrm{C}$ family and, as an important regulator of $\mathrm{p53}$, is responsible for the termination of the DDR and cellular signals for genotoxic stress $(4,5)$. Wip1 was originally identified as a target of the p53 protein; it is encoded by the protein phosphatase, $\mathrm{Mg}^{2+} / \mathrm{Mn}^{2+}$ dependent 1D (PPM1D) gene and activated upon genotoxic stress (6). Although Wip1 was initially identified as a nuclear phosphatase induced by $\mathrm{p} 53$ activation, subsequent studies have demonstrated that other transcription factors, including estrogen receptor $\alpha, \mathrm{c}-\mathrm{jun}$, cAMP response element-binding protein, $\mathrm{E} 2 \mathrm{~F}$ and $\mathrm{NF}-\kappa \mathrm{B}$, may induce the activation of Wip1 $(7,8)$. Once activated, Wip1 
negatively regulates p53 activation, either by directly dephosphorylating it or by activating E3 ubiquitin-protein ligase Mdm2, a p53 antagonist. The targets dephosphorylated by Wip1 also include ATM, H2A histone family member X (H2AX), Chk1, Chk2 and p38/MAPK, the key elements of the DDR (9). Wip1 is now recognized as an oncogenic phosphatase, as it has been found to be mutated, amplified and overexpressed in several types of human cancers harboring wt p53 (10-13). Oncogenic Wipl essentially leads to the suppression of key elements of the DDR and prevents the activation of genotoxic stress-induced cellular responses, such as checkpoint activation, DNA repair, apoptosis and senescence (14). The functions of Wip1 have been extensively studied in human solid tumors harboring functional p53 (15). Thus, Wip1 has emerged as a potential chemotherapeutic target in solid tumors that may increase p53-mediated anticancer responses. However, there are limited data evaluating its potential role in hematological cancers, particularly in those having an impaired p53 function. Therefore, in the present study, the role of Wip1 in the regulation of chemotherapy-induced cellular responses in the p53 mutant (mt) human ALL Jurkat cell line was investigated.

\section{Materials and methods}

Cell culture and drug treatments. The human T-cell acute lymphoblastic leukemia (T-ALL) cell line (Jurkat; ATCC ${ }^{\circledR}$ TIB-152 $2^{\mathrm{TM}}$ ), human breast cancer cell line (MCF-7; ATCC ${ }^{\circledR}$ HTB-22 ${ }^{\mathrm{TM}}$ ) and human foreskin fibroblasts (BJ; $\mathrm{ATCC}^{\circledR}$ CRL-2522 $2^{\mathrm{TM}}$ ) were obtained from the American Type Culture Collection. The Jurkat cells were cultured in RPMI-1640 medium supplemented with $10 \%(\mathrm{v} / \mathrm{v})$ fetal bovine serum (FBS), $100 \mathrm{IU} / \mathrm{ml}$ penicillin and $100 \mu \mathrm{g} / \mathrm{ml}$ streptomycin (all from Gibco; Thermo Fisher Scientific, Inc.). The MCF-7 and BJ cells were cultured in high glucose Dulbecco's modified Eagle's medium (DMEM), supplemented with 10\% FBS, $100 \mathrm{U} / \mathrm{ml}$ penicillin and $100 \mathrm{~g} / \mathrm{ml}$ streptomycin (all Gibco; Thermo Fisher Scientific, Inc.). All cells were maintained at $37^{\circ} \mathrm{C}$ in a humidified incubator with $5 \% \mathrm{CO}_{2}$. Doxorubicin and etoposide were obtained from Santa Cruz Biotechnology, Inc. DMSO was used as solvent control in the relevant experiments.

Genomic DNA isolation and gene copy number analysis by reverse transcription-quantitative $(R T-q) P C R$. Genomic DNA was isolated from Jurkat, MCF-7 and BJ cells using a GeneJET Genomic DNA Purification kit (Thermo Fisher Scientific, Inc.) according to the manufacturer's instructions. TaqMan gene copy number assays, namely PPM1D Gene Copy Number 20X (Assay ID: Hs05485469_cn) and Ribonuclease P (RNase P) Gene Copy Number Reference assay 20X (cat. no. 4403326) were from Applied Biosystems (Thermo Fisher Scientific, Inc.) and were used for qPCR analysis. RNase P served as the reference gene. The thermocycling conditions were as follows as: 10 -min hot start $\left(95^{\circ} \mathrm{C}\right)$, followed by 40 cycles of 2 -step qPCR with denaturing for $15 \mathrm{sec}$ at $95^{\circ} \mathrm{C}$ and annealing and extension for $1 \mathrm{~min}$ at $60^{\circ} \mathrm{C}$. The known control sample (gDNA from BJ fibroblasts carrying 2 alleles) was included in each reaction plate. All qPCR analyses were performed in triplicate using gDNA according to the manufacturer's protocol with a StepOne ${ }^{\mathrm{TM}}$
Real-Time PCR System (Applied Biosystems; Thermo Fisher Scientific, Inc.). The $2 \mathrm{X}$ relative copy number was calculated using StepOne Software V2.3 (Applied Biosystems; Thermo Fisher Scientific, Inc.) according to the $2^{-\Delta \Delta \mathrm{Cq}}$ comparative $\mathrm{Cq}$ method (16).

$R N A$ extraction and $R T-q P C R$. Total RNA was extracted from Jurkat, MCF-7 and BJ cells using a GeneJet RNA Purification kit (Thermo Fisher Scientific, Inc.). Complementary DNA (cDNA) was synthesized from the RNA using a High-Capacity cDNA Reverse Transcription kit (Applied Biosystems; Thermo Fisher Scientific, Inc.). according to the manufacturer's instructions. Taqman probes for the PPM1D gene (Assay ID: Hs01013292_m1) and internal control $\beta$-actin (Assay ID: Hs01060665_g1) were used from commercial assay kits (Applied Biosystems; Thermo Fisher Scientific, Inc.). The thermocycling conditions were as follows: $10 \mathrm{~min}$ at $95^{\circ} \mathrm{C}$, followed by 40 cycles at $95^{\circ} \mathrm{C} 15 \mathrm{sec}$ for denaturing and $1 \mathrm{~min}$ at $60^{\circ} \mathrm{C}$ for annealing and extension. Relative mRNA levels were calculated using StepOne Software V2.3 according to the $2^{-\Delta \Delta \mathrm{Cq}}$ comparative Cq method (16).

Cell viability assay. Cell viability assays were performed using the water soluble tetrazolium salt WST-1 (Sigma-Aldrich; Merck KGaA). Jurkat cells were incubated at $37^{\circ} \mathrm{C}$ for 24 and $72 \mathrm{~h}$ in the absence or presence of $1 \mu \mathrm{g} / \mathrm{ml}$ doxorubicin or $5 \mu \mathrm{g} / \mathrm{ml}$ etoposide. At the end of the incubation period, $10 \mu \mathrm{l}$ WST-1 solution was added to each well and the cells were incubated for $2 \mathrm{~h}$ at $37^{\circ} \mathrm{C}$ in a humidified incubator with $5 \% \mathrm{CO}_{2}$. The absorbance (A) was read at $450 \mathrm{~nm}$ using a Multiskan spectrum microplate reader (Thermo Labsystems). Cell viability was calculated according to the following formula: Viability $(\%)=\left[\left(\mathrm{A}_{\text {sample }}-\mathrm{A}_{\text {blank }}\right) /\left(\mathrm{A}_{\text {control }}-\mathrm{A}_{\text {blank }}\right)\right] \times 100$.

Small interfering RNA(siRNA) and transfection. siRNA oligonucleotides targeting the PPM1D gene (cat. no. sc-39205) and a control scrambled siRNA oligonucleotide (cat. no. sc-37007) were purchased from Santa Cruz Biotechnology, Inc. Lipofectamine ${ }^{\circledR} 2000$ (Invitrogen; Thermo Fisher Scientific, Inc.) was used for the transfection of Jurkat cells. In brief, $2.5 \times 10^{6}$ cells/well were cultured overnight at $37^{\circ} \mathrm{C}$ in $2.5 \mathrm{ml}$ complete RPMI-1640 without antibiotics in 12-well plates. Then, transfection was conducted at $37^{\circ} \mathrm{C}$ for $4 \mathrm{~h}$ using 40 pmol siRNA/well and Lipofectamine 2000 according to the manufacturer's protocol. At $16 \mathrm{~h}$ post transfection, cells were treated with appropriate drugs for indicated time points and subsequently used for Annexin V/7-amino-actinomycin (7AAD), caspase-3/7 activity, cell cycle and Ser-139 phosphorylated $\mathrm{H} 2 \mathrm{AX}(\gamma \mathrm{H} 2 \mathrm{AX})$ analysis or protein extraction.

Determination of apoptosis using caspase-3/7 and Annexin V/7AAD assays. Jurkat cells were incubated for 24 or $72 \mathrm{~h}$ in the absence or presence of $1 \mu \mathrm{g} / \mathrm{ml}$ doxorubicin or $5 \mu \mathrm{g} / \mathrm{ml}$ etoposide. The apoptosis profiles of the cells were determined using Muse ${ }^{\circledR}$ Annexin V and Dead Cell and Muse Caspase-3/7 kits (Merck KGaA) according to the manufacturer's instructions. Quantitative analyses of total apoptotic cells or active caspase-3/7-positive cells were performed using the Muse Cell Analyzer (Merck KGaA). 
Cell cycle analysis. A Muse Cell Cycle Assay kit (Merck $\mathrm{KGaA}$ ) was used to analyze of the cell cycle according to the manufacturer's instructions. In brief, cells were treated with the aforementioned concentrations of doxorubicin or etoposide for 24 or $72 \mathrm{~h}$ in triplicate. For senescence assays, cells were treated with lower concentrations $(0.2 \mu \mathrm{g} / \mathrm{ml}$ doxorubicin or $1 \mu \mathrm{g} / \mathrm{ml}$ etoposide) for $72 \mathrm{~h}$. At the end of the incubation period, cells were harvested, washed with $1 \mathrm{X}$ PBS and then fixed in $70 \%$ ethanol overnight. Following this, the cells were treated with $200 \mu \mathrm{l}$ Muse Cell Cycle reagent and the cell cycle distribution profiles were determined using the Muse Cell Analyzer.

Quantitative H2AX assay. Cells were treated in triplicate with $0.2 \mu \mathrm{g} / \mathrm{ml}$ doxorubicin or $1 \mu \mathrm{g} / \mathrm{ml}$ etoposide for $72 \mathrm{~h}$. At the end of the incubation period, cells were harvested, washed with 1X PBS and then assayed for the detection of $\gamma \mathrm{H} 2 \mathrm{AX}$. This was conducted using a Muse H2A.X Activation Dual Detection Assay (Merck KGaA) according to the manufacturer's instructions. Cells were analyzed using the Muse Cell Analyzer to quantify activated $(\gamma \mathrm{H} 2 \mathrm{AX})$ and inactivated (unphosphorylated) H2AX.

Protein extraction and western blot analysis. After drug treatment and/or transfection, cells were lysed in RIPA lysis buffer (150 mM NaCl, 5 mM EDTA pH 8.0, $50 \mathrm{mM}$ Tris $\mathrm{pH} 8.0,1 \% \mathrm{NP}-40,0.5 \%$ sodium deoxycholate and $0.1 \%$ SDS) supplemented with protease inhibitor cocktail (cOmplete $^{\mathrm{TM}}$; Roche Applied Science) and $1 \mathrm{mM} \mathrm{Na} \mathrm{VO}_{4}$. Total protein concentration was determined using a BCA assay according to the manufacturer's instructions (Thermo Fisher Scientific, Inc.) and $100 \mathrm{mg}$ protein/lane was loaded and separated by SDS-PAGE using 4-15\% Mini-PROTEAN ${ }^{\circledR}$ TGX $^{\mathrm{TM}}$ Precast Protein Gels (Bio-Rad Laboratories, Inc.) and electro-transferred onto PVDF membranes (Bio-Rad Laboratories, Inc.). The membranes were blocked in 5\% non-fat dried milk dissolved in 1X TBS-Tween for $1 \mathrm{~h}$ at room temperature and then incubated with primary antibodies at $4^{\circ} \mathrm{C}$ overnight and with secondary antibodies at room temperature for $1 \mathrm{~h}$ as previously described (17). Mouse anti-Wip1 (F-10; cat. no. sc-376257; 1:250), mouse anti-Chk1 (G-4; cat. no. sc-8408; 1:250), mouse anti-Chk2 primary antibodies (cat. no. sc-17747; 1:250) and horseradish peroxidase-coupled anti-mouse (cat. no. sc-2357; 1:1,000) or anti-rabbit (cat. no. sc-2004; 1:1,000) secondary antibodies were purchased from Santa Cruz Biotechnology, Inc. Polyclonal rabbit anti-phosphorylated (p)-Chk1 (S345; cat. no. 2348), polyclonal rabbit anti-p-Chk2 (T68; cat. no. 2197), monoclonal mouse anti-ATM (cat. no. 2873), rabbit anti-p-ATM (S1981; cat. no. 5883), monoclonal mouse anti-ATR (cat. no. 2790), polyclonal rabbit anti-p-ATR (S428; cat. no. 2853) antibody and polyclonal rabbit anti-H2AX (cat. no. 7631) and anti- $\gamma \mathrm{H} 2 \mathrm{AX}$ (S139; cat. no. 9718) were purchased from Cell Signaling Technology, Inc. (all 1:1,000). Monoclonal mouse anti-GAPDH (1:5,000; cat. no. 60004-1-Ig) was acquired from ProteinTech Group, Inc. Clarity Western ECL reagent (Bio-Rad Laboratories, Inc.) was used to visualize the bands, and a ChemiDoc-ItR2 Digital Imager (Analytik Jena AG) was used to image and analyze the membranes.
Senescence-associated (SA) $\beta$-galactosidase activity assay. Jurkat cells that were either untransfected or $16 \mathrm{~h}$ post transfection with siRNA were incubated in the absence or presence of $0.2 \mu \mathrm{g} / \mathrm{ml}$ doxorubicin or $1 \mu \mathrm{g} / \mathrm{ml}$ etoposide for at $37^{\circ} \mathrm{C}$ $72 \mathrm{~h}$, and the staining protocol was performed as described previously (17). Following staining photographs were captured with a digital camera under an inverted microscope (Leica Microsystems $\mathrm{GmbH}$ ). Cells were counted and the percentage of SA- $\beta$-gal-positive (blue) cells in the total cell population was calculated.

Statistical analysis. Origin 8.0 software (OriginLab Corporation) was used to calculate the mean and standard deviations of the results from at least three independent experiments each with three replicates. Statistical analysis was performed using one-way ANOVA followed by Bonferroni's multiple comparisons tests. $\mathrm{P} \leq 0.05$ was considered to indicate a statistically significant difference.

\section{Results}

Jurkat cells gain PPM1D amplification and express increased levels of Wipl phosphatase. In order to examine the role of Wip1 in hematological cancers, the Jurkat T-ALL cell line, which harbors mt p53, was used (18). In human cancers, oncogenic Wipl is amplified and overexpressed without exposure to genotoxic stress (15). Therefore, the present study initially examined whether the PPM1D gene is amplified and/or its expression is increased in Jurkat cells by measuring the gene copy number and mRNA levels using RT-qPCR. The MCF-7 cell line was used as a positive control, recognized for PPM1D/Wip1 gene amplification and overexpression (19). In addition, BJ human normal diploid fibroblasts were used as a negative control. As shown in Fig. 1A, the relative gene copy number of the PPM1D gene in Jurkat cells was significantly ( $\sim 10$-fold) higher than that in diploid BJ fibroblasts. The MCF-7 cells exhibited a higher copy number and mRNA level compared with the BJ and Jurkat cells (Fig. 1A). The relative mRNA level of Wip1 in Jurkat cells was significantly ( 4-fold) higher than that in BJ cells, but lower than that in MCF-7 cells (Fig. 1B). These results demonstrate that Jurkat cells harbor PPM1D amplification and express upregulated levels of Wip1 mRNA without genotoxic stress.

Etoposide and doxorubicin induce cell cycle arrest at the G2/M phase and decrease the viability of Jurkat cells. Subsequently, whether increased Wip1 expression affects the induction of genotoxic stress-mediated cellular responses in p53 mt Jurkat cells in a similar manner to that in wt p53 tumors was investigated. The DNA-damaging anticancer agents etoposide and doxorubicin were used to induce genotoxic stress and the viability of the Jurkat cells was then examined. As shown in Fig. 2A, following exposure to etoposide or doxorubicin for $24 \mathrm{~h}$, a slight but significant reduction in cell viability was observed, whereas exposure for $72 \mathrm{~h}$ caused a strong and significant reduction in viability (Fig. 2A). In addition, the proportion of cells in different cell cycle phases after treatment with etoposide or doxorubicin was examined. As shown in Figs. 2B and S1, $24 \mathrm{~h}$ of treatment with etoposide or doxorubicin induced only a slight increase in the proportion 

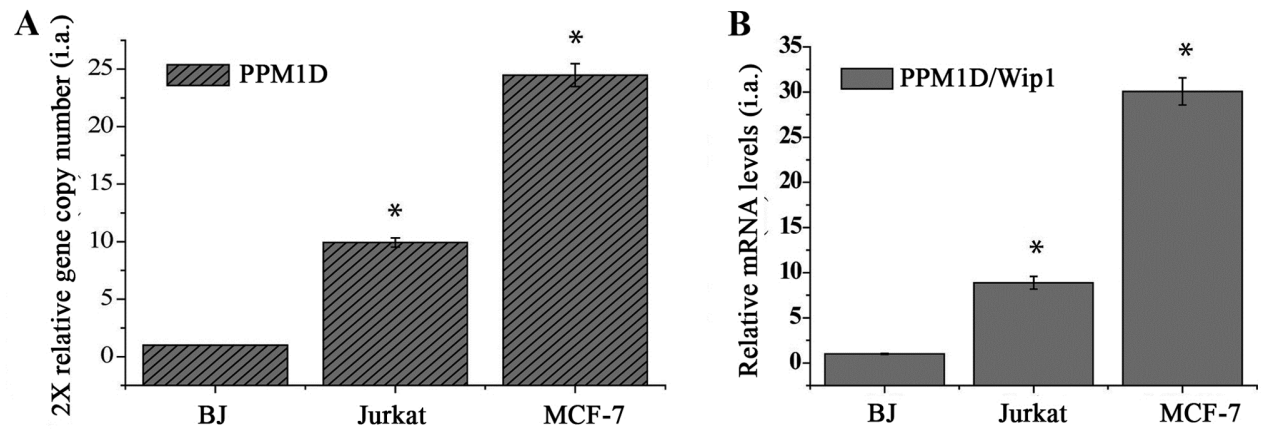

Figure 1. Jurkat cells exhibit PPM1D/Wip1 gene amplification and increased expression of Wip1. (A) Genomic DNA was isolated from BJ human diploid fibrobasts, Jurkat T-cell acute lymphoblastic leukemia cells and MCF-7 breast cancer cells and used for subsequent PPM1D gene copy number analysis by qPCR. RNAse P was used as the reference gene. (B) BJ, Jurkat and MCF7 cells were used for total RNA isolation. Relative Wip1 mRNA levels were analyzed by reverse transcription-qPCR. Data shown are the means \pm SD of three independent experiments. The statistical significance of differences in data was analyzed using one-way ANOVA followed by Bonferroni multiple comparisons tests. ${ }^{*} \mathrm{P} \leq 0.001 \mathrm{vs}$. BJ. PPM1D, protein phosphatase, $\mathrm{Mg}^{2+} / \mathrm{Mn}^{2+}$ dependent $1 \mathrm{D}$; Wip1, p53-induced phosphatase 1; qPCR, quantitative PCR.

A

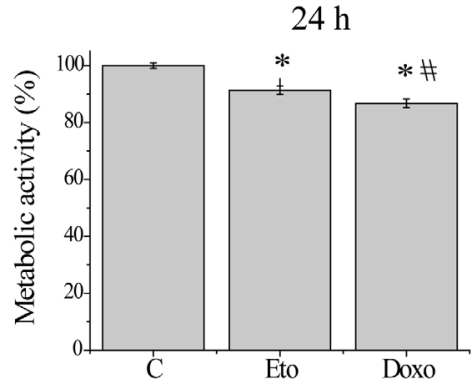

B

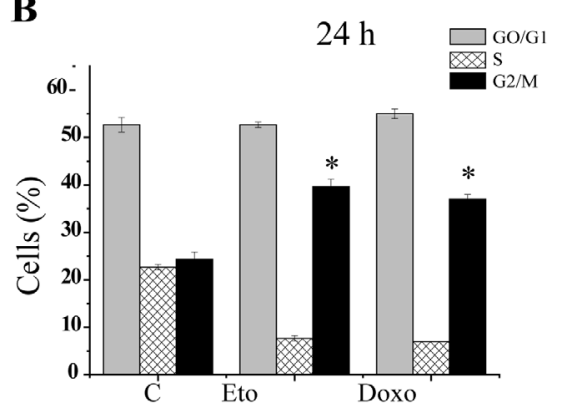

$72 \mathrm{~h}$
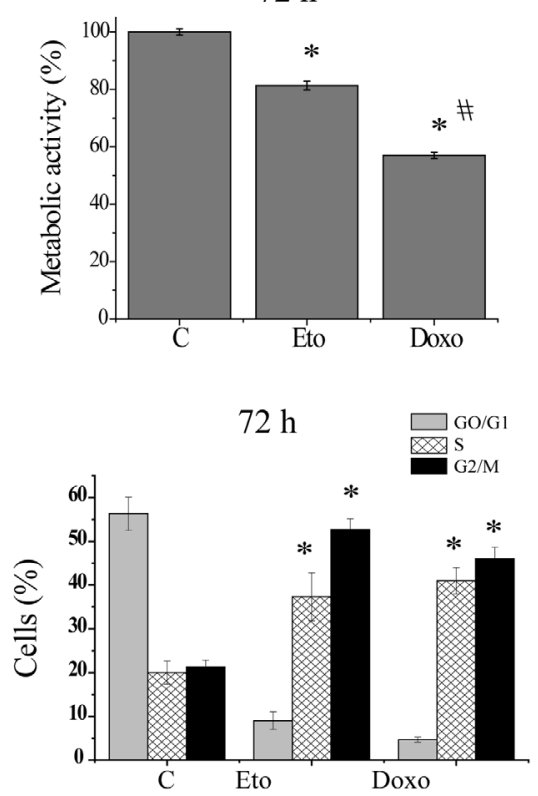

Figure 2. Eto and Doxo induce G2 cell cycle arrest and cell death. Jurkat cells treated with DMSO, $1 \mu \mathrm{g} / \mathrm{ml}$ Doxo or $5 \mu \mathrm{g} / \mathrm{ml}$ Eto for $24 \mathrm{~h}$ or 72 were subjected to (A) WST-1 assay and (B) cell cycle analysis. The percentage of Jurkat cells in the G0/G1, S and G2/M phases was calculated using a Muse Cell Analyzer. Data shown are the means \pm SD of three independent experiments. The statistical significance of differences in the data was analyzed using one-way ANOVA followed by Bonferroni multiple comparison tests. "P $\leq 0.001$ vs. C; ${ }^{~} \mathrm{P} \leq 0.001$ vs. Eto. C, DMSO control; Doxo, doxorubicin; Eto, etoposide.

of cells in the $\mathrm{G} 2$ phase, whereas $72 \mathrm{~h}$ of treatment strongly induced the accumulation of cells in the $\mathrm{S}$ and $\mathrm{G} 2 / \mathrm{M}$ phases. These results suggest that upregulated Wip1 expression did not prevent cell cycle arrest or the induction of cell death in Jurkat cells in response to etoposide or doxorubicin.

Etoposide and doxorubicin induce the apoptosis of Jurkat cells. Whether the decreased cell viability observed following treatment with etoposide or doxorubicin was due to the induction of an apoptotic response was then examined. As determined by Annexin V/7AAD analysis, treatment with etoposide or doxorubicin for $24 \mathrm{~h}$ induced a modest increase in apoptosis compared with that in the untreated control (Figs. 3A and S2A). By contrast, $72 \mathrm{~h}$ of treatment with etoposide or doxorubicin induced a significant increase in apoptosis to $27 \pm 1$ and $41 \pm 3 \%$, respectively, in Jurkat cells
(Figs. 3A and S2A). Similar results were also obtained using the caspase-3/7 activity assay. Following $24 \mathrm{~h}$ of treatment of the cells with etoposide or doxorubicin, only a small increase in the amount of active caspase-3/7 was observed, whereas $72 \mathrm{~h}$ of treatment induced a strong and significant increase (Figs. 3B and S2B). In addition, the phosphorylation status and total expression levels of known targets of Wip1, namely ATM, ATR, Chk1, Chk2 and H2AX, were analyzed. Upon treatment with etoposide or doxorubicin for $72 \mathrm{~h}$, the total protein levels of ATM, ATR, Chk1 and Chk2 were not markedly altered, and only weak phosphorylation levels of ATM (S1981), Chk1 (S345) and Chk2 (T68) were detectable. The phosphorylation of ATR was not detectable (data not shown). Notably, the phosphorylation of $\gamma \mathrm{H} 2 \mathrm{AX}$ (S139) was increased in response to treatment with etoposide or doxorubicin (Fig. 3C). 


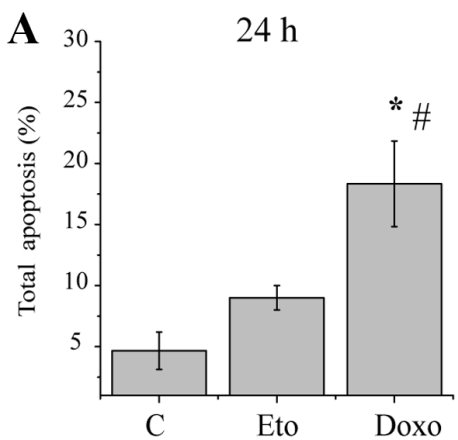

\section{B}

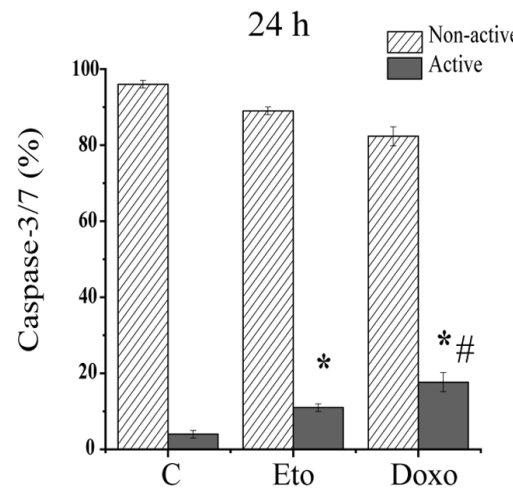

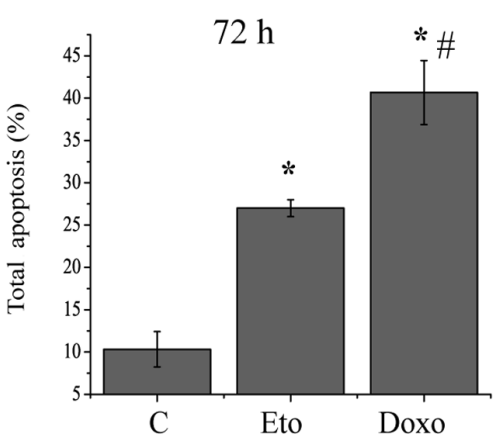

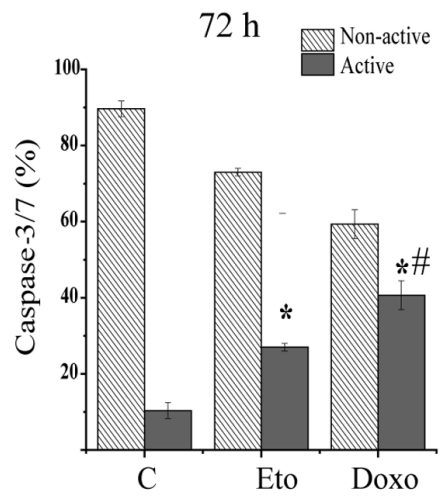

C
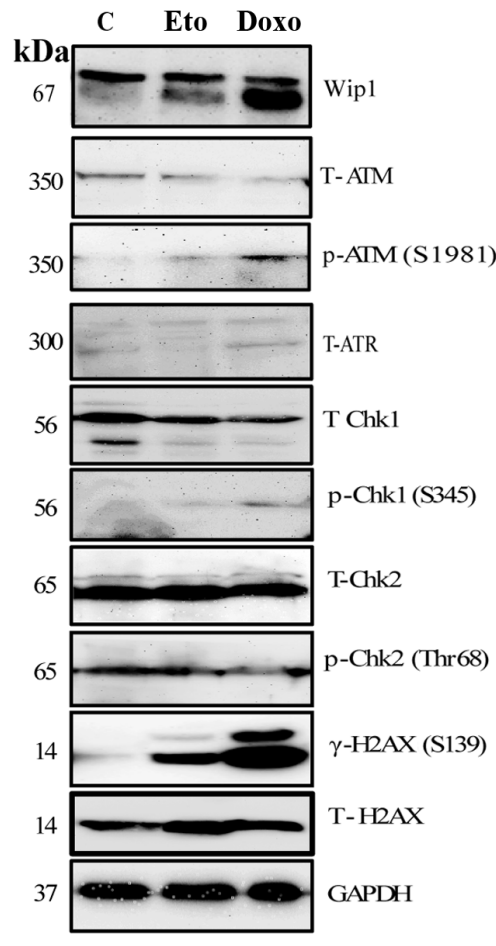

Figure 3. DNA damage response signaling is impaired in Jurkat cells, but the induction of apoptosis is retained. Jurkat cells treated with DMSO, $1 \mu \mathrm{g} / \mathrm{ml}$ Doxo or $5 \mu \mathrm{g} / \mathrm{ml}$ Eto for $24 \mathrm{~h}$ or $72 \mathrm{~h}$ were analyzed for (A) apoptosis using Annexin V/7AAD and (B) caspase-3/7 activity using a Muse Cell Analyzer, and (C) subjected to the western blot analysis of Wip1, p-ATM, T-ATM, ATR, p-Chk1, T-Chk1, p-Chk2, T-Chk2, gH2AX and H2AX. GAPDH was used as the loading control. Data shown are the means \pm SD of three independent experiments. The statistical significance of differences in the data was analyzed using one-way ANOVA followed with Bonferroni multiple comparison tests. ${ }^{*} \mathrm{P} \leq 0.001$ vs. C; ${ }^{\mathrm{P}} \leq 0.001$ vs. Eto. C, DMSO control; Doxo, doxorubicin; Eto, etoposide; Wip1, p53-induced phosphatase 1; p-, phospho-; T-, total; ATM, ataxia-telangiectasia mutated; ATR, ataxia-telangiestasia and Rad3-related; Chk, checkpoint kinase; H2AX, H2A histone family member X; gH2AX, p-H2AX (S139).

Etoposide and doxorubicin induce senescence in Jurkat cells. Since oncogenic Wipl is known to be involved in the negative regulation of senescence in response to DNA damage (15), the present study examined whether Jurkat cells are capable of undergoing senescence in response to etoposide or doxorubicin, despite the increased Wip1 activity. In order to avoid the induction of apoptosis, lower concentrations of etoposide or doxorubicin were used for the induction of senescence. Accordingly, the effects of the lower concentrations of etoposide or doxorubicin on cell viability were examined. As shown in Fig. 4A, no significant reductions in cell viability occurred following the two treatments and the majority of cells were viable. One of the hallmarks of senescence is cell cycle arrest; hence, the cell cycle distribution of Jurkat cells following $72 \mathrm{~h}$ of exposure to the lower concentrations of etoposide and doxorubicin was examined. As shown in Fig. 4B, etoposide or doxorubicin treatment led to the marked and significant arrest of Jurkat cells in the G2 phase of the cell cycle (Figs. 4B and S3A). In addition, the levels of the phosphorylated protein $\gamma \mathrm{H} 2 \mathrm{AX}$, which is another senescence marker, were measured. As shown in Figs. $4 \mathrm{C}$ and S3B, the $\gamma \mathrm{H} 2 \mathrm{AX}$ levels were significantly increased in response to $72 \mathrm{~h}$ of etoposide or doxorubicin treatment. In addition, the etoposide- or doxorubicin-treated cells were stained for detection of the senescence marker SA- $\beta$-galactosidase. Jurkat cells were positive for SA- $\beta$-galactosidase activity, indicating that both etoposide and doxorubicin induced senescence (Fig. 4D). Since the induction of senescence is mediated by DDR signaling, the present study examined whether key elements of the DDR signaling pathway were phosphorylated. The results were similar to those for apoptosis; weak levels of p-ATM (S1981), p-Chk1 (S345) and p-Chk2 (T68) were detectable, whereas the total levels of the proteins were not altered (Fig. 4E).

\section{Knockdown of Wip1 restores DDR signaling, but decreases} apoptosis. To gain further insight into the function of Wip1 in p53 mt Jurkat cells, Wip1 was knocked down and the cellular responses were further examined. The expression of Wip1 protein was markedly decreased following the siRNA-mediated knockdown of Wipl (Fig. 5A). Key elements of DDR signaling were examined, and western blotting revealed that the knockdown of Wipl clearly increased the phosphorylation of ATM (S1981), Chk1 (S345), Chk2 (T68) and ATR (S428) in the absence of genotoxic stress. A slight increase in the total protein levels of ATR, Chk1 and Chk2 was also visible compared with the respective levels in the cells transfected with control siRNA (Fig. 5A). Whether the knockdown of Wipl affected the cell cycle status of Jurkat cells in response to etoposide or doxorubicin treatment was then determined. Notably, no significant changes were detected in the proportions of cells in different cell cycle stages when Wip1 was knocked down. Etoposide or doxorubicin induced G2 cell cycle arrest in a similar manner to that in the control cells, regardless of whether the cells were transfected with control siRNA 
A

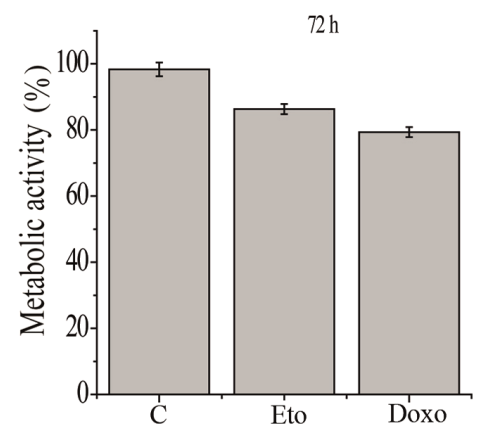

B

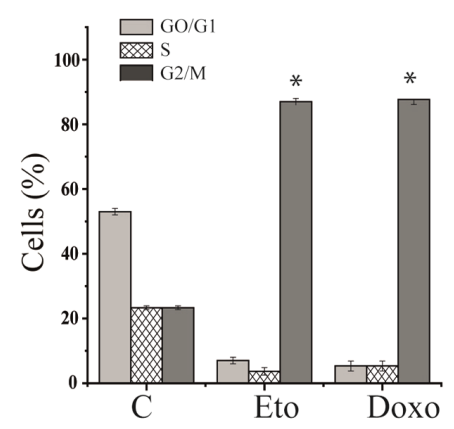

C

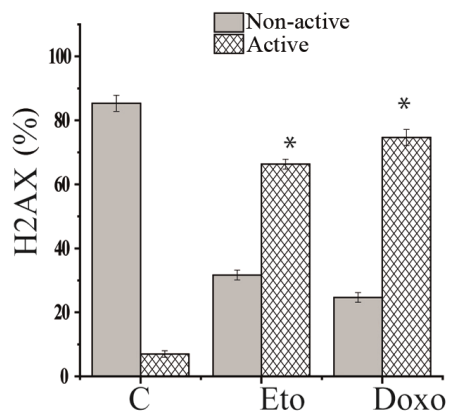

D

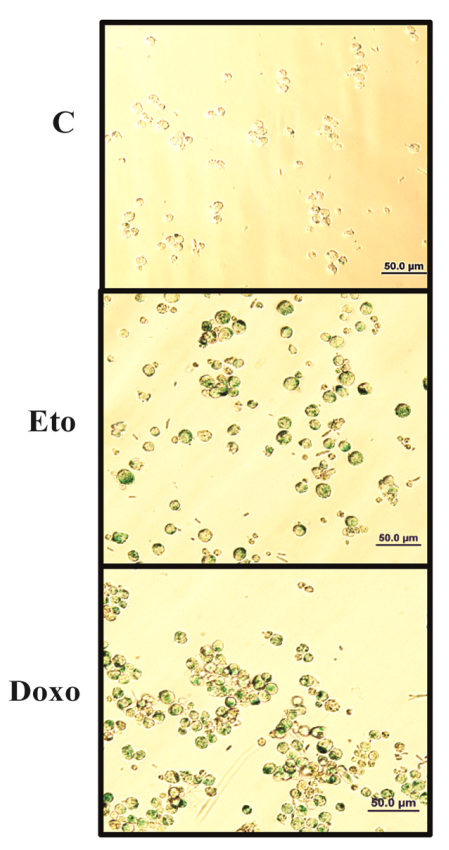

E $\quad$ kDa C Eto Doxo

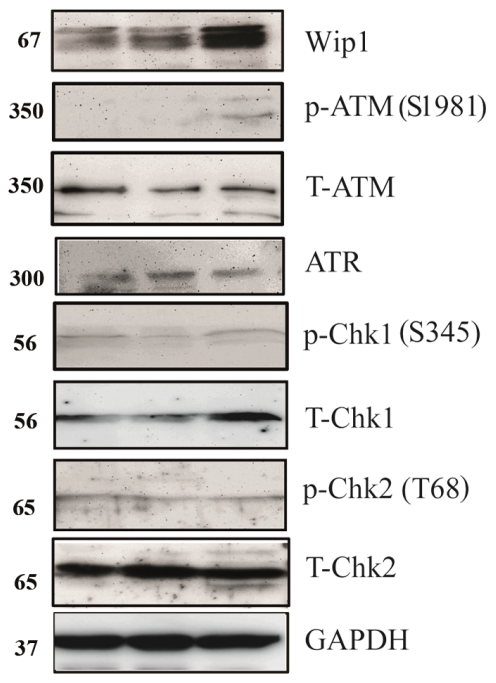

Figure 4. Induction of senescence is unaffected by increased Wip1 in Jurkat cells. Jurkat cells were treated with DMSO, $0.2 \mu \mathrm{g} / \mathrm{ml}$ Doxo or $1 \mu \mathrm{g} / \mathrm{ml}$ Eto for $72 \mathrm{~h}$ and the induction of senescence was assayed by measuring (A) cell viability by WST-1 assay, (B) the cell cycle profile and (C) the amount of active H2AX [ $\gamma \mathrm{H} 2 \mathrm{AX} ; \mathrm{p}-\mathrm{H} 2 \mathrm{AX}$ (S139)] and non-active (unphosphorylated) H2AX. (D) Staining of cells for senescence-associated $\beta$-galactosidase activity. Scale bar, $50 \mathrm{~mm}$. (E) Western blot analysis of Wip1, p-ATM, T-ATM, ATR, p-Chk1, T-Chk1, p-Chk2 and T-Chk2. GAPDH was used as the loading control. Data shown are the means $\pm \mathrm{SD}$ of three independent experiments. The statistical significance of differences in the data was analyzed by one-way ANOVA followed by Bonferroni multiple comparison tests. "P $\leq 0.001$ vs. C. C, DMSO control; Doxo, doxorubicin; Eto, etoposide; H2AX, H2A histone family member X; Wip1, p53-induced phosphatase 1; p-, phospho-; T-, total; ATM, ataxia-telangiectasia mutated; ATR, ataxia-telangiestasia and Rad3-related; Chk, checkpoint kinase.

or siRNA targeting Wip1 (Figs. 5B and S4). Subsequently, the effect of the knockdown of Wipl on the apoptosis induced by etoposide or doxorubicin was evaluated. The results demonstrated that the levels of apoptosis induced by etoposide or doxorubicin were significantly decreased when Wip1 was knocked down compared with those in the cells transfected with control siRNA (Figs. 5C and S5A). The amount of total apoptosis was decreased by $\sim 10 \%$ in response to etoposide and doxorubicin treatment when Wipl was knocked down. Similar data were obtained in the caspase-3/7 activity assay. The knockdown of Wipl significantly decreased the amount of activated caspase-3/7 induced in response to etoposide or doxorubicin treatment (Figs. 5D and S5B).

Knockdown of Wipl does not promote the induction of senescence. The effect of knocking down Wip1 on senescence and cell cycle arrest in response to etoposide or doxorubicin treatment in Jurkat cells was investigated. As shown in Figs. 6A and S6A, the knockdown of Wip1 did not alter the cell cycle status of Jurkat cells in response to etoposide or doxorubicin. No differences in the proportion of cells in each cell cycle phase between the Wip1 siRNA- or control siRNA-transfected cells were observed (Fig. 6A). In addition, the phosphorylation of $\gamma \mathrm{H} 2 \mathrm{AX}$ was measured quantitatively. Notably, following the knockdown of Wip1, $72 \mathrm{~h}$ of exposure to etoposide or doxorubicin increased the levels of the phosphorylated protein $\gamma \mathrm{H} 2 \mathrm{AX}$ (Figs. 6B and S6B). The SA $\beta$-galactosidase activity of Jurkat cells in response to etoposide or doxorubicin treatment was also evaluated when Wip1 was knocked down. The results revealed that the cells in which Wip1 was knocked down were capable of undergoing senescence, and no significant changes were detected in the SA $\beta$-galactosidase positivity of the cells (Fig. 6C). These results suggest that the knockdown of Wipl activity restores DDR signaling and decreases apoptosis, but does not affect the cell cycle or senescence of p53 mt Jurkat cells. 
A

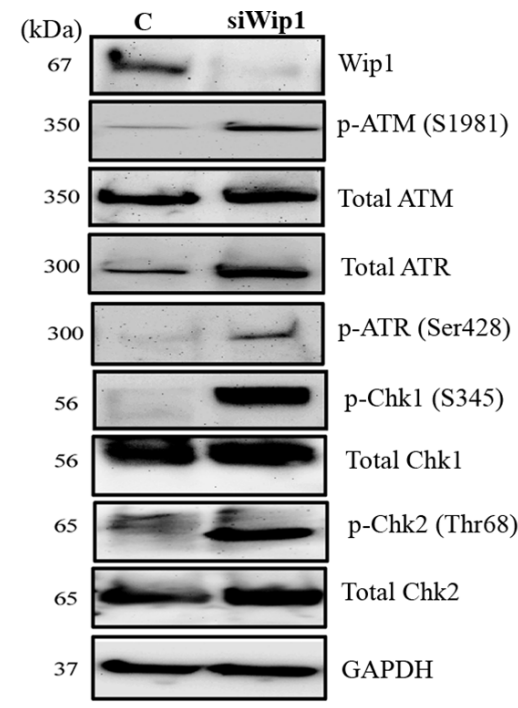

B

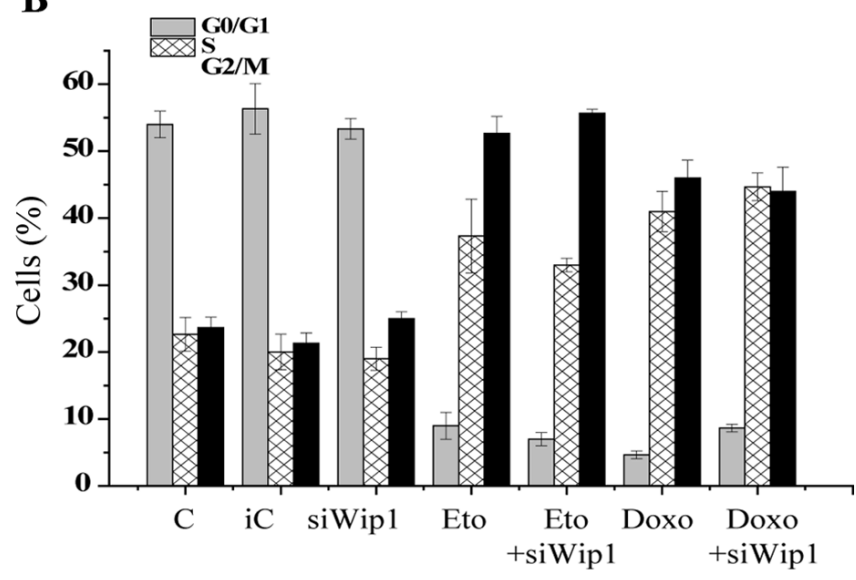

C

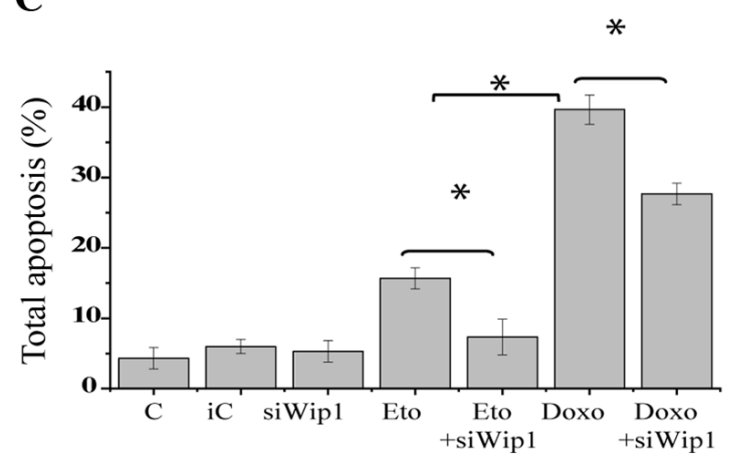

D

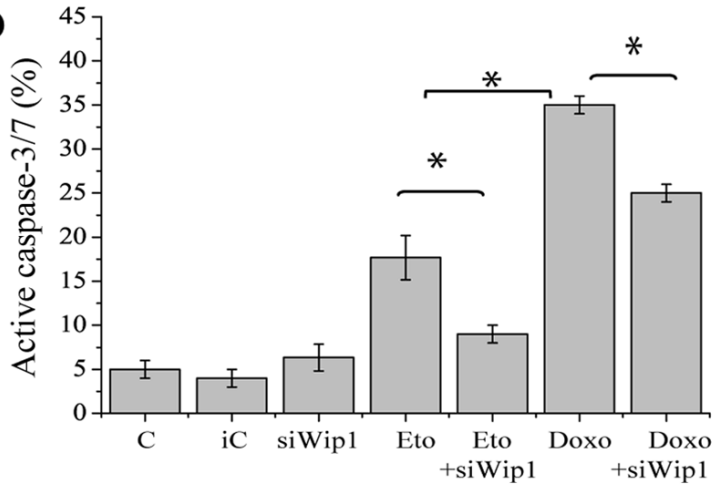

Figure 5. Knockdown of Wip1 restores DNA damage response signaling but decreases apoptosis. Jurkat cells were transfected with siWip1 or siC siRNA. (A) The cells were subjected to western blot analysis of Wip1, p-ATM, T-ATM, p-ATR, T-ATR, p-Chk1, T-Chk1, p-Chk2 and T-Chk2. GAPDH was used as the loading control. siRNA. The transfected cells were treated with DMSO, $1 \mu \mathrm{g} / \mathrm{ml}$ Doxo or $5 \mu \mathrm{g} / \mathrm{ml}$ Eto for $72 \mathrm{~h}$ and subjected to (B) cell cycle analysis (C) Annexin/7AAD staining for apoptosis analysis and (D) caspase-3/7 activity assay. Data are expressed the as means \pm SD of three independent experiments. The statistical significance of differences in the data was analyzed using one-way ANOVA followed by Bonferroni multiple comparison tests. "P $\leq 0.001$ as indicated. Wip1, p53-induced phosphatase 1; siWip1, siRNA targeting Wip1; siC, inverted siRNA control that does not target any gene; siRNA, small interfering RNA; C, DMSO control; Doxo, doxorubicin; Eto, etoposide; p-, phospho-; T-, total; ATM, ataxia-telangiectasia mutated; ATR, ataxia-telangiestasia and Rad3-related; Chk, checkpoint kinase.

\section{Discussion}

The function of the p53 tumor suppressor gene is impaired in more than half of human cancers (20), whereas in cancers harboring wt $\mathrm{p} 53$, mechanisms have developed to bypass the tumor suppressor function of p53 (21). The increased activity of serine threonine phosphatase Wip1 is recognized as one of the mechanisms by which the functions of p53 associated with genotoxic stress responses are bypassed (22). In healthy cells, upon genotoxic stress, Wip1 is activated and provides feedback inhibition to p53 that terminates DDR signaling. By contrast, Wip1 is amplified in cancer cells; it is upregulated and acts as an oncogene, suppressing DDR and p53 activation. Oncogenic Wipl has been extensively studied and is accepted as a therapeutic target in human solid tumors harboring functional p53 (15). However, its function in p53-impaired tumors has rarely been evaluated (23). Thus, the present study aimed to evaluate the role of Wip1 in the regulation of chemotherapy-induced cellular responses in the p53 mt human ALL cell line Jurkat (18). To the best of our knowledge, the present study is the first to demonstrate that p53 mt Jurkat cells exhibit PPM1D gene amplification and increased expression of Wip1 phosphatase. The increased Wip1 expression enhances the apoptosis and senescence sensitivity of Jurkat cells by attenuating DDR signaling and dephosphorylating ATM, ATR, Chk1 and Chk2. By contrast, the knockdown of Wip1 restores DDR signaling, but decreases the sensitivity of Jurkat cells to chemotherapeutic agents.

This conclusion was confirmed by several lines of evidence. First, evidence was provided by gene copy number and gene expression analysis, which demonstrated that Jurkat cells exhibit PPM1D amplification and express high levels of Wip1 mRNA in the absence of genotoxic stress. Second, cell cycle analysis demonstrated that etoposide and doxorubicin each caused a significant increase in the accumulation of cells in the $\mathrm{S}$ and $\mathrm{G} 2 / \mathrm{M}$ phases. More importantly, it demonstrated that the cell cycle status of Jurkat cells in response to etoposide or doxorubicin treatment was not altered by the knockdown of Wip1. In a previous study on neural progenitor cells, it was observed that Wip1 did not influence the cell cycle status of 
A

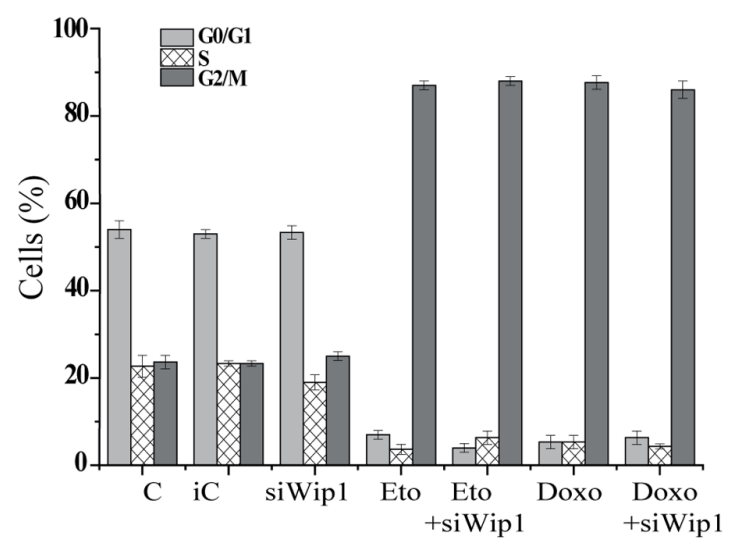

B

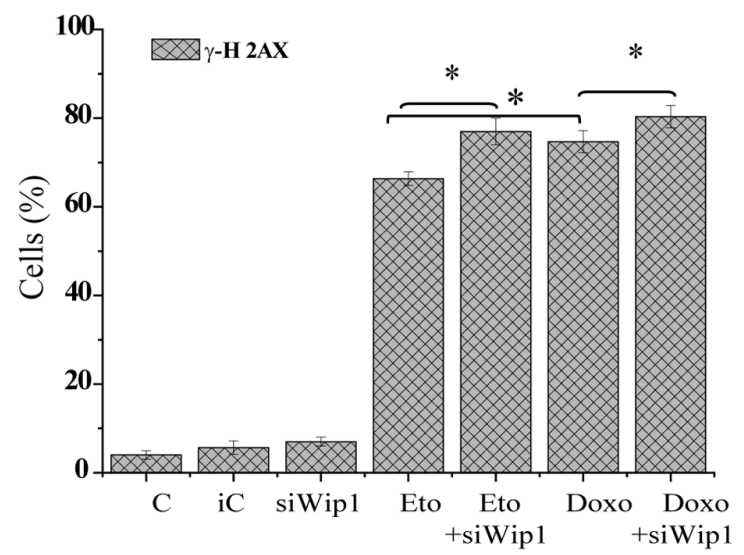

C Eto Doxo
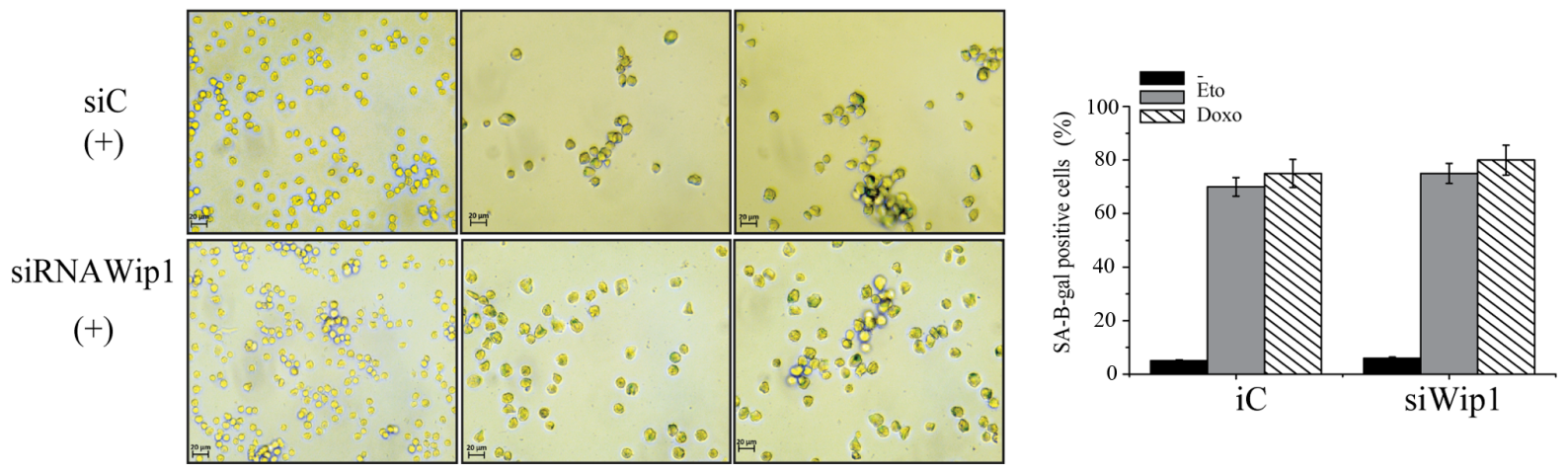

Figure 6. Knockdown of Wip1 does not affect cell cycle or senescence. (A) Jurkat cells were transfected with siWip1 or siC siRNA and treated with DMSO, $0.2 \mu \mathrm{g} / \mathrm{ml}$ Doxo or $1 \mu \mathrm{g} / \mathrm{ml}$ Eto for $72 \mathrm{~h}$ and induction of senescence was assayed by measuring the cell cycle profile. (B) Quantitative $\gamma \mathrm{H} 2 \mathrm{AX}$ assay and (C) senescence-associated $\beta$-galactosidase activity staining and quantification were also performed. Scale bar, $20 \mathrm{~mm}$. Data are shown as the means \pm SD of three independent experiments. The statistical significance of differences in the data were analyzed using one-way ANOVA followed by Bonferroni multiple comparison tests. "P $\leq 0.001$ as indicated. Wip1, p53-induced phosphatase 1; siWip1, siRNA targeting Wip1; siC, inverted siRNA control that does not target any gene; siRNA, small interfering RNA; C, DMSO control; Doxo, doxorubicin; Eto, etoposide.

p53 knockout neural progenitor cells, but it did affect it in p53 wt cells (24). Furthermore, another study reported that Wip1 did not affect the cell cycle distribution of p53- or p21-knockout-MCF7 cells, suggesting that the effects of Wip1 on cell cycle distribution depend mainly on p53 and p21 activity (13). Hence, the findings of the present study are in line with previous research.

An important finding of the present study is that upregulated expression of Wip1 enhances the apoptotic sensitivity of Jurkat cells to etoposide and doxorubicin treatment. This was confirmed by Annexin V/7AAD and caspase-3/7 activity measurements, which showed that the knockdown of Wip1 significantly decreased the levels of apoptosis in response to etoposide or doxorubicin. Previous studies have demonstrated that targeting Wip1 enhances sensitivity to chemotherapy and promotes apoptosis in human cancers harboring wt p53. However, contradictory data have been reported for p53-deficient tumors $(23,25)$. For example, the study conducted by Goloudina et al (23) demonstrated that the inhibition of Wip1 increased the sensitivity of wt p53 HCT116 colon cancer cells to cisplatin-induced apoptosis, but did not have such an effect on p53-/- HCT116 or Saos-2 cells. The study also demonstrated that in response to anticancer drugs, Wip1 overexpression promoted apoptosis via the induction of Bax through activation of the transcription factor RUNX2 in cells with inactive p53. These data suggest that Wip1 functions as a sensitization factor to anticancer drugs in p53 mt Jurkat cells.

The present study provides evidence that the activation of DDR signaling is attenuated in Jurkat cells in response to etoposide or doxorubicin. Therefore, it is suggested that the increased expression of Wip1 phosphatase in Jurkat cells may be involved in the suppression of DDR signaling. This hypothesis was confirmed by the results demonstrating that the knockdown of Wipl increased the phosphorylation of ATM, ATR, Chk1 and Chk2, even in the control cells without any genotoxic stress.

Another noteworthy finding of the present study is that $\gamma \mathrm{H} 2 \mathrm{AX}$ levels were increased, although the phosphorylation status of other targets of Wip1, namely ATM, ATR, Chk1 and Chk2, was not altered in response to etoposide or doxorubicin treatment. Previous studies have demonstrated that Wip1 targets and dephosphorylates key elements of the DDR, including ATM, ATR, Chk1 and Chk2 kinases, as well as $\gamma \mathrm{H} 2 \mathrm{AX}$. The phosphorylation of H2AX on serine 139 is induced in response to DNA damage and is designated as 
$\gamma \mathrm{H} 2 \mathrm{AX}$. The phosphorylation of $\mathrm{H} 2 \mathrm{AX}$ to form $\gamma \mathrm{H} 2 \mathrm{AX}$ has been specifically recognized as a marker for the generation of DNA double-strand breaks (26). Generally, ATM kinase is considered to be the main physiological mediator of the phosphorylation of H2AX in response to DNA damage. However, studies have suggested that H2AX can also be phosphorylated by other phosphoinositide 3-kinase-associated protein kinases, including ATR and/or DNA-dependent protein kinase (DNA-PKc) (27,28). Thus, since the present study did not detect any marked phosphorylation of ATM or ATR, it is possible that other kinases, such as DNA-PKc, may be responsible for the increased phosphorylation of $\gamma \mathrm{H} 2 \mathrm{AX}$ in response to etoposide or doxorubicin treatment. The data demonstrating that the knockdown of Wip1 significantly increased the phosphorylation of ATM and ATR in control cells, but not the phosphorylation of H2AX in a similar proportion, support the hypothesis.

In healthy cells, the DDR maintains the integrity of the genome and defects in the DDR result in damaged DNA being unrepaired, which ultimately leads to the accumulation of mutations, genomic instability and cancer initiation. Indeed, previous research has indicated that deficiencies in the DDR frequently occur in human cancers. However, DDR defects also provide targetable susceptibilities that are relatively specific to cancer cells, which may be exploited for clinical benefit with the use of DDR inhibitors (29). In line with previous findings, the present study demonstrated that the knockdown of Wip1 increased DDR activity, but decreased apoptotic sensitivity, also suggesting that apoptotic resistance may be due to increased DDR signaling and DNA repair, as previously reported (29). However, based on the current findings that the knockdown of Wip1 increased the phosphorylation of ATM, ATR, Chk1 and Chk2 without any genotoxic stress, it may be deduced that the increased expression of Wipl is an early event occurring during the tumorigenesis of Jurkat cells in order to bypass the DDR.

DNA damage-induced senescence is known as another important cellular response, besides apoptosis, that is activated by chemotherapeutic agents (30). In particular, treatment with sublethal concentrations of conventional DNA-damaging anticancer agents readily induces premature senescence in cancer cells $(23,30)$. Previous research has demonstrated the negative effects of oncogenic Wipl on the induction of senescence by chemotherapeutic agents in wt p53 cell lines (13). However, other research has provided contrasting data, suggesting that Wip1 phosphatase is downregulated during persistent DNA damage and p53-dependent senescence. The inhibitory effects of Wip1 on the induction of senescence are mostly dependent on p53 and p21 activity (31). The present study provides evidence that, despite the increased expression of Wip1, etoposide and doxorubicin induced senescence in p53 mt Jurkat cells. Furthermore, the induction of senescence was not affected by the knockdown of Wip1, and Wip1 was not downregulated during senescence. In Jurkat cells, the induction of senescence was independent of p53 and, thus, Wip1 was ineffective in this response.

In conclusion, the present study demonstrated that p53 mt Jurkat cells exhibit amplification of the PPM1D gene and the upregulated expression of Wip1 phosphatase. The increased expression of Wip1 attenuates DDR signaling by dephosphorylating ATM, ATR, Chk1 and Chk2; however, the ability of chemotherapeutic agents to induce apoptosis and senescence is retained. Thus, the present study, to the best of our knowledge, is the first to demonstrate that increased Wipl expression enhances the sensitivity of the p53 mt ALL cell line to chemotherapy-induced apoptosis, unlike the effect observed in solid tumors with wt p53. The present study highlights the importance of careful consideration when devising future treatment strategies that aim to manipulate or target Wip1, as targeting Wip1 in human cancers lacking p53 may not yield the same results as in tumors harboring wt p53.

\section{Acknowledgements}

Not applicable.

\section{Funding}

The present study was supported by Aydın Adnan Menderes University, Scientific Research Projects Foundation (grant no. TPF1-16029) and The Scientific and Technological Research Council of Turkey (TUBITAK; grant no. 214S200).

\section{Availability of data and materials}

All data generated or analyzed during this study are included in this published article.

\section{Authors' contributions}

MKE designed the study, performed the experiments, analyzed the data, wrote the manuscript, provided funding and obtained the grants. NBK and HP performed the experiments and analyzed the data. NBK and MKE confirm the authenticity of all the raw data. All authors have read and approved the final manuscript.

\section{Ethics approval and consent to participate}

Not applicable.

\section{Patient consent for publication}

Not applicable.

\section{Competing interests}

The authors declare that they have no competing interests.

\section{Authors' information}

The ORCIDs of the authors are as follows: Mehtap Kilic Eren, https://orcid.org/0000-0003-3811-9819; Nur Betül Kartal, https://orcid.org/0000-0003-1750-1672; Hatice Pilevneli, https://orcid.org//0000-0003-1455-7283.

\section{References}

1. Bartek J, Bartkova J and Lukas J: DNA damage signalling guards against activated oncogenes and tumour progression. Oncogene 26: 7773-7779, 2007. 
2. Halazonetis TD, Gorgoulis VG and Bartek J: An oncogene-induced DNA damage model for cancer development Science 319: 1352-1355, 2008.

3. Jackson SP and Bartek J: The DNA-damage response in human biology and disease. Nature 461: 1071-1078, 2009.

4. Shimada M and Nakanishi M: Response to DNA damage: Why do we need to focus on protein phosphatases? Front Oncol 3: 8 , 2013.

5. Wang ZP, Tian Y and Lin J: Role of wild-type p53-induced phosphatase 1 in cancer. Oncol Lett 14: 3893-3898, 2107.

6. Fiscella M, Zhang HL, Fan S, Sakaguchi K, Shen S, Mercer EW, Vande Woude GF, O'Connor MP and Appella E: Wip1, a nove human protein phosphatase that is induced in response to ionizing radiation in a p53-dependent manner. Proc Natl Acad Sci USA 94: 6048-6053, 1997.

7. Chew J, Biswas S, Shreeram S, Humaidi M, Wong ET, Dhillion MK, Teo H, Hazra A, Fang CC, López-Collazo E, et al WIP1 phosphatase is a negative regulator of NF-kappaB signalling. Nat Cell Biol 11: 659-666, 2009

8. Le Guezennec X and Bulavin DV: WIP1 phosphatase at the crossroads of cancer and aging. Trends Biochem Sci 35: 109-114, 2010.

9. Lowe J, Cha H, Lee MO, Mazur SJ, Appella E and Fornace AJ Jr: Regulation of the Wipl phosphatase and its effects on the stress response. Front Biosci (Landmark Ed) 17: 1480-1498, 2012

10. Zhao M, Zhang H, Zhu G, Liang J, Chen N, Yang Y, Liang X, Cai $\mathrm{H}$ and Liu W: Association between overexpression of Wipl and prognosis of patients with non-small cell lung cancer. Oncol Lett 11: 2365-2370, 2016

11. Goloudina AR, Kochetkova EY, Pospelova TV and Demidov ON: Wip1 phosphatase: Between p53 and MAPK kinases pathways. Oncotarget 7: 31563-31571, 2016.

12. Yin S, Wang P, Yang L, Liu Y, Wang Y, Liu M, Qi Z, Meng J, Shi TY, Yang G and Zang R: Wipl suppresses ovarian cancer metastasis through the ATM/AKT/Snail mediated signaling. Oncotarget 7: 29359-29370, 2016.

13. Pechackova S, Burdova K, Benada J, Kleiblova P, Jenikova G and Macurek L: Inhibition of WIP1 phosphatase sensitizes breast cancer cells to genotoxic stress and to MDM2 antagonist nutlin-3. Oncotarget 7: 14458-14475, 2016.

14. Macurek L, Benada J, Müllers E, Halim VA, Krejčíková K, Burdová K, Pecháčková S, Hodný Z, Lindqvist A, Medema RH and Bartek J: Downregulation of Wip1 phosphatase modulates the cellular threshold of DNA damage signaling in mitosis. Cell Cycle 12: 251-262, 2013.

15. Pecháčková S, Burdová K and Macurek L: WIP1 phosphatase as pharmacological target in cancer therapy. J Mol Med (Berl) 95: 589-599, 2017

16. Livak KJ and Schmittgen TD: Analysis of relative gene expression data using real-time quantitative PCR and the 2(-Delta Delta C(T)) method. Methods 25: 402-408, 2001.

17. Eren MK and Tabor V: The role of hypoxia inducible factor- 1 alpha in bypassing oncogene-induced senescence. PLoS One 9: e101064, 2014.

18. Drexler HG, Fombonne S, Matsuo Y, Hu ZB, Hamaguchi $\mathrm{H}$ and Uphoff CC: $p 53$ alterations in human leukemia-lymphoma cell lines: In vitro artifact or prerequisite for cell immortalization? Leukemia 14: 198-206, 2000.

19. Bulavin DV, Demidov ON, Saito S, Kauraniemi P, Phillips C, Amundson SA, Ambrosino C, Sauter G, Nebreda AR, Anderson CW, et al: Amplification of PPM1D in human tumors abrogates p53 tumor-suppressor activity. Nat Genet 31: 210-215, 2002 .
20. Martin AC, Facchiano AM, Cuff AL, Hernandez-Boussard T, Olivier M, Hainaut $\mathrm{P}$ and Thornton JM: Integrating mutation data and structural analysis of the TP53 tumor-suppressor protein. Human Mutat 19: 149-164, 2002.

21. Bond GL, Hu W and Levine AJ: MDM2 is a central node in the p53 pathway: 12 years and counting. Curr Cancer Drug Targets 5: 3-8, 2005.

22. Lu X, Ma O, Nguyen TA, Jones SN, Oren M and Donehower LA The Wip1 Phosphatase acts as a gatekeeper in the p53-Mdm2 autoregulatory loop. Cancer Cell 12: 342-54, 2007.

23. Goloudina AR, Tanoue K, Hammann A, Fourmaux E, Le Guezennec X, Bulavin DV, Mazur SJ, Appella E, Garrido C and Demidov ON: Wip1 promotes RUNX2-dependent apoptosis in p53-negative tumors and protects normal tissues during treatment with anticancer agents. Proc Natl Acad Sci USA 109: E68-E75, 2012

24. Zhu YH, Zhang CW, Lu L, Demidov ON, Sun L, Yang L, Bulavin DV and Xiao ZC: Wipl regulates the generation of new neural cells in the adult olfactory bulb through p53-dependent cell cycle control. Stem Cells 27: 1433-1442, 2009.

25. Xia ZS, Wu D, Zhong W, Lu XJ, Yu T and Chen QK: Wip1 gene silencing enhances the chemosensitivity of human colon cancer cells. Oncol Lett 14: 1875-1883, 2017.

26. Burma S, Chen BP, Murphy M, Kurimasa A and Chen DJ: ATM phosphorylates histone $\mathrm{H} 2 \mathrm{AX}$ in response to DNA double-strand breaks. J Biol Chem 276: 42462-42467, 2001.

27. Mukherjee B, Kessinger C, Kobayashi J, Chen BP, Chen DJ, Chatterjee A and Burma S: DNA-PK phosphorylates histone $\mathrm{H} 2 \mathrm{AX}$ during apoptotic DNA fragmentation in mammalian cells. DNA Repair 5: 575-590, 2006.

28. Wang H, Wang M, Wang H, Böcker W and Iliakis G: Complex $\mathrm{H} 2 \mathrm{AX}$ phosphorylation patterns by multiple kinases including ATM and DNA-PK in human cells exposed to ionizing radiation and treated with kinase inhibitors. J Cell Physiol 202: 492-502, 2005.

29. Pilié PG, Tang C, Mills GB and Yap TA: State-of-the-art strategies for targeting the DNA damage response in cancer. Nat Rev Clin Oncol 16: 81-104, 2019.

30. Kilic M and Schmitt CA: Exploiting drug induced senescence in transgenic mouse models. In: Beyond Apoptosis: Cellular Outcomes of Cancer Therapy. Roninson IB, Brown JM and Bredesen DE (eds). Informa Health Care Publication, pp273-295, 2008.

31. Crescenzi E, Raia Z, Pacifico F, Mellone S, Moscato F, Palumbo G and Leonardi A: Down-regulation of wild-type p53-induced phosphatase 1 (Wip1) plays a critical role in regulating several p53-dependent functions in premature senescent tumor cells. J Biol Chem 288: 16212-24, 2013.

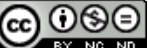

This work is licensed under a Creative Commons Attribution-NonCommercial-NoDerivatives 4.0 International (CC BY-NC-ND 4.0) License. 